\title{
SwissDRG: 3 Jahre alt und schon Routine?
}

\section{Pierre-François Cuénoud}

Dr. med., Vizepräsident der FMH, Departementsverantwortlicher Tarife und Gesundheitsökonomie Spitalärzte

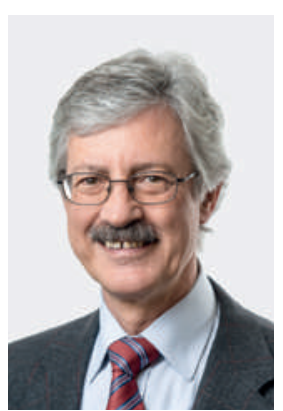

Die Tarifstruktur SwissDRG, die vor mehr als 1100 Tagen ins Leben gerufen wurde, liegt mittlerweile bereits in ihrer 4 . Version vor. Dem Wunsch der Partner, eine hinreichende Dynamik in die Struktur zu bringen, um möglichst schnell die sachgerechte $\mathrm{Ab}$ bildung der stationären Spitalleistungen zu gewährleisten, wurde entsprochen. Dank der ausgezeichneten Arbeit des Case Mix Office (CMO) hat sich der Anspruch, ein spezielles gesamtschweizerisches Tarifsystem zu schaffen und fortlaufend weiterzuentwickeln, als realistisch erwiesen. Die Reaktionsfähigkeit dieses Systems ist gut; das Kostengewicht kann von einer Version zur nächsten um bis zu 30 Prozent schwanken.

Natürlich ist das System nicht in jeder Hinsicht optimal. Besonders Hochkostenfälle stellen für die Universitäts- und Zentrumsspitäler weiterhin eine Belastung dar. Sowohl das politische Klima als auch die Stimmung in der Bevölkerung sind den allgemeinen Anliegen der öffentlichen Gesundheit aktuell sehr zuträglich, was sich indirekt auch in der Tarifstruktur für die Spitäler niederschlägt; so wurden die Leistungen der Grundversorgung zulasten bestimmter technischer und komplexerer Leistungen schrittweise bessergestellt. Dieser Umstand verzögert aber spürbar das Erreichen eines Systems, das ausgereift genug ist, um eine korrekte Abgeltung der Leistungen anstreben zu können, ohne die Baserate je nach Art der Einrichtung differenzieren zu müssen.

Der Wechsel zum SwissDRG ist erfolgreich verlaufen. Natürlich ist die Situation nicht in jeder Hinsicht optimal: Spitalaufenthalte, die mit sehr hohen Kosten verbunden sind, stellen für die Universitäts- und Zentrumsspitäler weiterhin eine Belastung dar.

Für die Patienten haben sich in diesen drei Jahren, in denen die Fallpauschalen zur Anwendung kamen, keine offenkundigen Änderungen ergeben. Für die Spitalleitungen hingegen gestaltet sich die Situation häufig schwierig. Die Verhandlungen mit den Versicherern laufen regelmässig auf eine Senkung der Baserate hinaus, wodurch die Entscheidungsträger gezwungen werden, bestimmte Leistungen abzubauen, um das finanzielle Gleichgewicht zu wahren. Um die adäquate Gesundheitsversorgung ihrer jeweiligen Bevölkerung dauerhaft sicherzustellen, müssen die Kantone die gemeinwirtschaftlichen Leistungen übernehmen: Einige Kantone gewähren jedoch keine einzige dieser Leistungen und zwingen damit ihre Spitäler zu überlebenssichernden Verschlankungsmassnahmen. Andere Kantone wiederum zeigen sich überaus grosszügig und lassen ihren öffentlichen Einrichtungen jährlich hunderte Millionen zukommen oder räumen ihnen Sonderkredite für die Erneuerung ihrer Infrastruktur ein. Dies führt landesweit zu einer erheblichen Ungleichbehandlung aller Beteiligten.

Trotz der zunehmenden Bürokratisierung vernachlässigen die Spitalärztinnen und -ärzte keinesfalls ihre klinischen Aufgaben.

Trotz der zunehmenden Bürokratisierung vernachlässigen die Spitalärztinnen und -ärzte keinesfalls ihre klinischen Aufgaben. Allerdings ist hinsichtlich der Sensibilität eine allmähliche Veränderung zu beobachten: Während sich noch vor zehn Jahren nur einer von zwanzig Kaderärzten für wirtschaftliche Belange interessierte, gibt heute ein Drittel an, sich aktiv an der Spitalverwaltung zu beteiligen; ein Grossteil hat entsprechende Weiterbildungsprogramme absolviert. Ausserdem geben immer mehr Kolleginnen und Kollegen ihre klinische Tätigkeit auf, um sich neuen, rein administrativen Berufsfeldern wie dem kürzlich eingeführten "Management von $\mathrm{Pa}$ tientenpfaden» zu widmen. Die Anzahl an Medizinern, die aufgrund dieser Verlagerung innerhalb eines Jahrzehnts für den Dienst am Patienten verlorengegangen sind, entspricht einem ganzen Absolventenjahrgang.

Insgesamt ist der Wechsel zu SwissDRG erfolgreich verlaufen. Mit kritischem Engagement setzt sich die FMH beständig für die Verbesserung der Tarifstruktur ein. Andererseits fungiert sie als Schnittstelle zwischen den medizinischen Anwendern und den Dachorganisationen. Sie stellt somit sicher, dass die Ärzteschaft ihren massgeblichen Einfluss auf unsere stationäre Gesundheitsversorgung bewahren kann, die zu viel Entwicklungspotential aufweist, um in Routine zu verfallen. 